$\delta 1890.0$ cioè $\delta_{0}$

\begin{tabular}{|c|c|c|c|c|c|c|c|c|}
\hline Sistema & $\tau$ Cygni & Oss. & 79 Cygni & Oss. & 12 Androm. & Oss. & P Androm. & Oes. \\
\hline $\begin{array}{l}\text { Bradley-Auwers } \\
\text { Respighi }\end{array}$ & $\begin{array}{lll}37^{\circ} & 34^{\circ} & 34^{\prime}: 27 \\
37 & 34 & 34.46\end{array}$ & $\begin{array}{l}11 \\
3^{8}\end{array}$ & 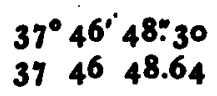 & $\begin{array}{c}4 \\
20\end{array}$ & $\begin{array}{l}37^{\circ} 34^{\circ} .54^{\prime \prime} 53 \\
37 \quad 34 \quad 55.43\end{array}$ & $\begin{array}{c}3 \\
20\end{array}$ & $\begin{array}{lll}37^{\circ} 21^{\prime} & 33^{\prime}: 42 \\
37 & 21 & 32.64\end{array}$ & 21 \\
\hline
\end{tabular}

Errore medio del complesso dei $\varphi-\delta_{0}= \pm 0.022$.

Errore medio del peso uno $= \pm 0.24$.

Latitudine nel sistema Bradley-Auwers $=3^{8^{\circ}} 6^{\prime} 44^{\prime \prime} 359$ con 40 osservazioni di $\delta_{0}$.

Latitudine nel sistema Respighi $=3^{8^{\circ}} 6^{\circ} 44^{\prime \prime} 594$ con 99 osservazioni di $\delta_{0}$.

Combinandole tenendo conto delle osservazioni di $\delta_{0}$ si ha $3^{\circ} 6^{\prime} 44^{\prime \prime} 5^{26}$; questa ridotta al cerchio di Ramsden conferma entro un decimo di secondo la latitudine del Piazzi che da un secolo circa si usa nel nostro osservatorio.

Palermo I891 gennaio 5 .

T. Zona.

\title{
Genäherte Elemente und Ephemeride des Cometen 1884 III (Wolf)
}

für seine Wiederkehr im Jahre 189 r.

Von A. Thraen, Pfarrer zu Dingelstaedt.

Bei der Berechnung der definitiven Bahnelemente des Cometen 1884 III (Wolf), welche ich aus den Beobachtungen seiner ersten Erscheinung 1884-85 abgeleitet und in Nr. 2789-90 der A. N. veröffentlicht habe, hatte ich die Störungen desselben unberïcksichtigt gelassen, weil ich sie von vornherein für unbedeutend hielt. Da aber Herr LehmannFilhés dieselben bei seiner Bestimmung der vormaligen Bahn des Cometen (A. N. 2953) vermisste, so habe ich nach. träglich die Coordinaten-Störungen durch Erde und Jupiter für die erste Erscheinung berechnet. Die für die zu Grunde liegenden Normalörter gefundenen Beträge der Störungen, ausgedrückt in Einheiten der 7. Decimale,

$$
\begin{array}{ccccccccc} 
& 1 & 2 & 3 & 4 & 5 & 6 & 7 & 8 \\
\xi^{\prime} & 0 & +1 & +6 & +15 & +23 & +20 & +2 & -24 \\
\eta^{\prime} & -1 & -3 & -17 & -58 & -128 & -223 & -373 & -520 \\
\zeta^{\prime} & -1 & -2 & -13 & -43 & -90 & -151 & -240 & -323
\end{array}
$$

waren merkbar genug, um zur nochmaligen Auflösung der l.c. angeführten Normalgleichungen zu veranlassen, nachdem neue Absolutglieder aus den Differenzen berechnet waren, welche sich nunmehr unter Anbringung der Störungen zwischen Beobachtung und Rechnung ergaben, nämlich:

$$
\begin{array}{ccccccccc} 
& 1 & 2 & 3 & 4 & 5 & 6 & 7 & 8 \\
\mathrm{~d} \alpha \cdot \cos \delta & +0.20 & -0.28 & +0.05 & +1.79 & +1.67 & +2.29 & +0.23 & +6.68 \\
\mathrm{~d} \delta & +0.11 & -0.05 & +0.47 & +0.49 & +0.65 & +1.57 & +3.97 & +2.45
\end{array}
$$

Durch die hieraus erlangten Verbesserungen $\mathrm{d} M=-12.82 ; \mathrm{d} \pi=+3.52 ; \mathrm{d} \Omega=+29.60 ; \mathrm{d} i=+0.30$; $\mathrm{d} q=-32.55 ; \mathrm{d} \mu=+0.25758 \mathrm{nahm}$ das auf 1890.0 bezogene Elementen-System folgende Gestalt an:

Osculation und Epoche 1884 October 4.0 M. Z. Berlin

$$
\begin{aligned}
& M=353^{\circ} 28^{\prime} 32^{\prime \prime} 35 \\
& \pi^{\prime}=165241.20 \\
& \left.\begin{array}{rrrr}
\delta^{\prime}=272 & 51 & 5.47 \\
i^{\prime}= & 10 & 58 & 14.60
\end{array}\right\} \begin{array}{c}
\text { Mittl. Aequator } \\
1890.0
\end{array} \\
& \varphi=34^{\circ} 6^{\prime} 4^{\prime \prime} .14 \\
& \mu=524.00227 \\
& \log a=0.5537823
\end{aligned}
$$

Bei der Darstellung der Normalörter mittelst dieser Elemente ergaben' sich folgende Differenzen zwischen Beobachtung und Rechnung:

$$
\begin{array}{ccccccccc} 
& \mathrm{I} & 2 & 3 & 4 & 5 & 6 & 7 & 8 \\
\mathrm{~d} \alpha \cdot \cos \delta & +0.39 & -0.17 & -0.28 & +0.95 & +0.63 & +0.63 & -2.79 & +1.82 \\
\mathrm{~d} \delta & -0.64 & +0.26 & +0.70 & -0.24 & -1.13 & -0.81 & +0.97 & -0.89
\end{array}
$$

$\mathrm{Da}$ die Elemente aus den in Nr. 2790 angegebenen Gründen noch mit beträchtlicher Unsicherheit behaftet sind, so schien es eine vergebliche Müe, für die Wiederkehr des Cometen im Jahre $189 \mathrm{I}$ schon jetzt eine genaue Ephemeride $2 \mathrm{u}$ berechnen. Um zu seiner Wieder-Auffindung eine genäherte Ephemeride herstellen zu können, habe ich darum mit obigen Elementen nur seine Störungen durch Erde und
Jupiter berechnet, zumal da er inzwischen anderen grossen Planeten nicht nahe kommt. Da er auch dem Jupiter beim diesmaligen Umlauf ziemlich fern bleibt, so schien für die Störungs-Rechnung das Intervall von 40 Tagen für den vorliegenden Zweck ausreichend. Ich behalte mir vor, genauere Störungs-Rechnungen, auch von den anderen Planeten, namentlich Mars und Saturn, nachzuholen, sobald nach seiner 
hoffentlichen Wieder-Auffindung die Bahnelemente, besonders die Excentricität und mittlere tägliche Bewegung, auf Grund der Beobachtungen seiner zweiten Erscheinung sicherer bestimmt sind. Nach Durchführung der Rechnungen für die
Störungen durch Erde und Jupiter nach der Methode der Variation der Constanten erhielt ich für 189 I Juli 10.0 folgende Störungs-Werthe, wobei die Ausgangs-Epoche 1884 Sept. 24.0 war.

$$
\begin{aligned}
& \begin{array}{llllllllll}
\text { Einfache Integrale } & 40 \Delta \mu & (\Delta L)_{1} & \Delta \pi & \Delta \varphi & \Delta \Omega & \Delta i & \text { Doppelintegral }
\end{array}
\end{aligned}
$$

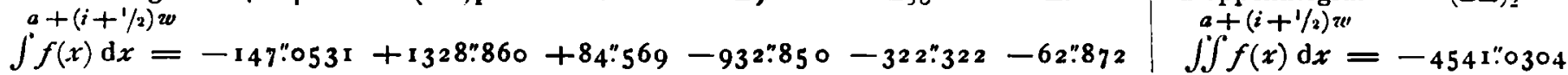

Nachdem an obige Elemente, welche zuvor auf die Ekliptik übertragen waren, vorstehende Beträge angebracht waren (wobei $\Delta \mu=-3.6763$ und $\Delta L=(\Delta L)_{1}+(\Delta L)_{2}=$ $-53^{\prime} 32^{\prime \prime} \mathrm{I} 7$; ferner $\mu_{0} . t=360^{\circ} 5^{\prime}{ }^{\prime} 45^{\prime \prime} 63$ wurde), entstand folgendes Elementen-System :

Osculation und Epoche 1891 Juli 10.0 M. Z. Berlin

$$
\begin{aligned}
& M=352^{\circ} 5^{\prime} \quad \text { I. } 2^{\prime \prime} \quad \text { oder } 35^{\circ} 5^{\prime} \text { I".2 } \\
& L=\quad \text { I I I } 5 \text { 50.8 } \\
& \pi=19 \text { 10 } 49.6 \\
& \delta=2062 \text { I } 40.4 \\
& i=251430.8 \\
& \varphi=335^{I} 9.3 \\
& \mu=520.3260 \\
& \log a=0.5558207
\end{aligned}
$$

Heliocentrische Aequatorealcoordinaten für 189 I.0.

$$
\begin{aligned}
& x=[9.9920635] r . \sin \left(v+106^{\circ} 5^{\prime} 34^{\prime \prime} 56\right) \\
& y=[9.9999803] r . \sin (v+165215.86) \\
& z=[9.278031 \mathrm{I}] r . \sin (v+104 \quad 251.45)
\end{aligned}
$$

Nach diesem System würde (abgesehen von den

\begin{tabular}{|c|c|c|c|c|c|c|c|c|}
\hline \multicolumn{2}{|c|}{1891} & \multicolumn{2}{|c|}{$\boldsymbol{\alpha}$} & \multicolumn{2}{|c|}{$\delta$} & \multirow{2}{*}{$\frac{\log r}{0.2533}$} & \multirow{2}{*}{$\frac{\log 4}{0.2590}$} & \multirow{2}{*}{$\frac{H}{2.7}$} \\
\hline Juni & 12 & $0^{b}$ & $5^{m} 14^{s}$ & $+22^{\circ}$ & $14: 3$ & & & \\
\hline & 16 & - I & 5 & +23 & 3.0 & $0.249 \mathrm{I}$ & 0.2482 & 2.9 \\
\hline & 20 & - 2 & 5 & +23 & 49.4 & $0.245^{\circ}$ & 0.2374 & 3.1 \\
\hline & 24 & & 515 & +24 & $33 \cdot 3$ & 0.2410 & 0.2264 & $3 \cdot 3$ \\
\hline & 28 & 04 & $153 x$ & +25 & I 4.4 & 0.2371 & 0.2153 & 3.6 \\
\hline Juli & $\begin{array}{l}2 \\
6\end{array}$ & $\begin{array}{ll}0 & 5 \\
1 & \end{array}$ & $\begin{array}{ll}5 & 55 \\
6 & 26\end{array}$ & $\begin{array}{l}+25 \\
+26\end{array}$ & $\begin{array}{l}52.3 \\
26.6\end{array}$ & $\begin{array}{l}0.2334 \\
0.2298\end{array}$ & $\begin{array}{l}0.2041 \\
0.1928\end{array}$ & $\begin{array}{l}3.8 \\
4.1\end{array}$ \\
\hline & 10 & I I & 4 & +26 & 57.1 & 0.2264 & 0.1813 & 4.4 \\
\hline & 14 & 12 & 748 & +27 & 23.3 & $0.223^{2}$ & 0.1697 & 4.7 \\
\hline & 18 & I 3 & 836 & +27 & 44.7 & $0.220 \mathrm{I}$ & 0.1580 & 5.0 \\
\hline & 22 & 14 & 1928 & +28 & 1.2 & 0.2173 & 0.1462 & 5.4 \\
\hline & 26 & & 022 & +28 & r 2.3 & 0.2147 & 0.1342 & 5.7 \\
\hline & 30 & $2 x$ & $1 \times 16$ & +28 & 17.9 & 0.2123 & 0.1221 & 6.1 \\
\hline Aug. & 3 & 22 & 128 & +28 & 16.9 & 0.2101 & 0.1100 & 6.5 \\
\hline & 7 & 23 & 256 & +28 & 9.6 & 0.2082 & 0.0978 & 7.0 \\
\hline & I I & 24 & 1337 & +27 & 55.5 & 0.2065 & 0.0854 & $7 \cdot 4$ \\
\hline & I 5 & 25 & 4410 & +27 & 34.4 & $0.205 \mathrm{I}$ & $0.073^{\circ}$ & 7.9 \\
\hline
\end{tabular}
weiteren Störungen) das Perihel stattfinden 189 I Sept. 2.77 105. Der Durchgang durch den niedersteigenden Knoten tritt Sept. I 4.5 ein. Die Umlaufszeit beträgt 2490.746 oder $U=6.82$ tropische Jahre.

Aus vorstehenden Elementen wurde folgende genäherte Ephemeride abgeleitet, wobei die Coordinaten-Störungen innerhalb derselben unberücksichtigt gelassen wurden, weiI sie nur zur Wieder-Auffindung dienen soll und sich nicht weit von der Osculations-Epoche entfernt.

Ephemeride für I $^{\text {h }}$ M. Z. Berlin.

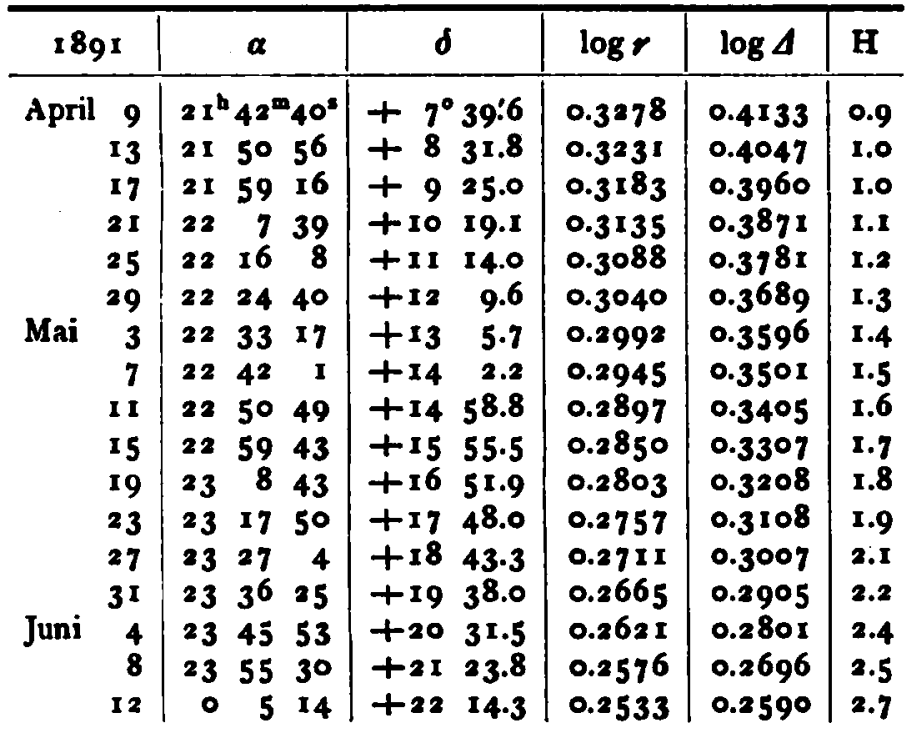

Als Einheit der theoretischen Helligkeit wurde die Lichtstärke gewählt, welche der Comet am Tage der letzten Beobachtung desselben - am 6. April 1885 - hatte. Dies wird beiläufig wieder der Fall sein am 13. April 1891, zu welcher Zeit die Ephemeride beginnt. Dieselbe beschränkt sich zunächst auf 4 Monate, weil unterdessen nach seiner Wieder-Auffindung voraussichtlich sich eine genauere Ephemeride wird herstellen lassen.

Um zu untersuchen, wie weit die Oerter der Ephemeride bei einer anderen Annahme für die unsichersten Elemente $\mu$ und $\varphi$ sich ändern würden, habe ich schliesslich mit denselben eine Variation vorgenommen. Bei der letzten Auflösung der Normalgleichungen waren die Incremente dieser beiden Elemente an einander geknüpft gewesen durch die Relation $y=[9.77249]+\left[9.63170_{n}\right] w$ und gefunden $y=[1.53007]$. Indem ich diesen Werth um das Fünffache variirte, also $y^{\prime}=[2.2904]$ setzte, wurde $w^{\prime}=\left[2.59582_{n}\right]$ und demzufolge $\mu^{\prime}=525.03257$ und $\varphi^{\prime}=34^{\circ} 4^{\prime} 29^{\prime \prime 64}$. Demnach wurde für I89 I Juli 10.0 $M^{\prime}=35^{\circ}{ }^{\circ} 47^{\prime} 3^{6.4}$ I $; \mu^{\prime}=521{ }^{\prime \prime} 35^{6} 3 ; \varphi^{\prime}=33^{\circ} 4^{\prime} 8^{\prime} 6^{\prime \prime} 79 ;$ $\log a^{\prime}=0.555^{2480}$. Die ubrigen Elemente, welche nur die Lage bestimmen, $\pi, \Omega, i$, wurden unverändert wie oben beibehalten. Hiermit wurde für einige Epochen folgende Ephemeride für $12^{\text {h }}$ M. Z. Berlin berechnet. 


\begin{tabular}{|c|c|c|c|c|c|c|}
\hline i $89 \mathrm{r}$ & $\alpha$ & $\delta$ & $\log r$ & $\log \Delta$ & $\alpha^{\prime}-a$ & $\delta^{\prime}-\delta$ \\
\hline April 2 I & $22^{\mathrm{h}} 14^{\mathrm{m}} 3^{8^{\mathrm{a}}}$ & $+10^{\circ} 28^{\prime} 1$ & & 77 & $+6: 98$ & $+8: 99$ \\
\hline Mai I I & $22 \quad 5^{8} \quad 5^{2}$ & +155.1 & 0.2837 & 0.3420 & +8.04 & $+63^{\circ}$ \\
\hline Mai 3I & $\begin{array}{lll}23 & 45 & 59\end{array}$ & +1936.5 & 0.2613 & 0.2945 & +9.57 & -1.42 \\
\hline Juni 20 & - $3^{6} 8$ & +2336.7 & 0.2399 & 0.2431 & +11.04 & -12.69 \\
\hline Juli ro & I 2944 & +2627.1 & 0.2223 & 0.1898 & +12.66 & -30.00 \\
\hline
\end{tabular}

In den Columnen $\left(\alpha^{\prime}-\alpha\right)$ und $\left(\delta^{\prime}-\delta\right)$ habe ich den Unterschied zwischen obiger Ephemeride und der entsprechenden Variation hinzugefügt. Die Veränderungen in AR. sind hiernach sehr beträchtlich. Die obige Annahme eines fünffachen Werthes für $y$ liegt aber auch ganz ausserhalb der Wahrscheinlichkeit.

Dingelstaedt (Eichsfeld) I89 I März 12.

A. Thraen. Pfarrer.

Zusatz. Herr A. Berberich in Berlin hat schon vor einiger Zeit aus seinen Elementen in A. N. 2800 mit Berücksichtigung der Jupiterstörungen bis 189 I eine Aufsuchungsephemeride für die nächsten Monate berechnet und Herrn Prof. Barnard auf der Lick-Sternwarte zugehen lassen. Zur Vergleichung mit der Thraen'schen Ephemeride erlaube ich mir einige Oerter nach Herrn Berberich's Rechnung (für $12^{\text {h }}$ M. Z. Berlin) mitzutheilen:

\begin{tabular}{|c|c|c|}
\hline 1891 & $\alpha$ & $\delta$ \\
\hline tril & $21^{\mathrm{h}} 41^{\mathrm{m}} 19^{\circ}$ & +7 \\
\hline 17 & $\begin{array}{lll}21 & 57 & 49 \\
22 & 14 & 35\end{array}$ & $\begin{array}{r}+922.8 \\
+1112.0\end{array}$ \\
\hline
\end{tabular}

\begin{tabular}{|c|c|c|}
\hline $189 r$ & $\boldsymbol{\alpha}$ & $\delta$ \\
\hline 0 & $22^{h} 31^{m} 40^{\circ}$ & $+13^{\circ} 3: 9$ \\
\hline & $\begin{array}{rrr}22 & 49 & 4 \\
23 & 6 & 51\end{array}$ & $\begin{array}{r}+14 \\
+167.4 \\
+1650.9\end{array}$ \\
\hline
\end{tabular}

\begin{tabular}{|c|c|c|}
\hline 1891 & $\alpha$ & $\delta$ \\
\hline $\begin{array}{lr}\text { Mai } & 27 \\
\text { Juni } & 4\end{array}$ & $\begin{array}{l}23^{\mathrm{h}} 25^{\mathrm{m}} 4^{\mathrm{s}} \\
234345\end{array}$ & $\begin{array}{l}+18^{\circ} 43^{\prime} 1 \\
+2032.0 \\
\text { Krueger. }\end{array}$ \\
\hline
\end{tabular}

\section{Ephemeride des Planeten (266) Aline.}

Den Planeten (266) hat man in seiner zweiten Erscheinung uberhaupt nicht und in der dritten nur dreimal in Wien beobachtet. Durch diese Beobachtungen wurden die Elemente in A. N. 2944 verbessert und es ergab sich folgendes Elementensystem :

Epoche und Osculation: 1887 Juni 2 I.O M. Z. Berlin.

$$
\begin{aligned}
& M=237^{\circ} 54^{\circ} 55^{\prime \prime} \mathrm{I} \\
& \omega=14755 \quad 57.1 \\
& \Omega=23^{6} \quad 21 \quad 42.2 \\
& i=\begin{array}{lll}
13 & 23 & 33.8
\end{array} \\
& \varphi=9^{\circ} 7^{\circ} 55^{\prime \prime 6} \\
& \mu=753^{\prime \prime 224} \\
& \log a=0.448722
\end{aligned}
$$

\begin{tabular}{|c|c|c|c|c|}
\hline $189 \mathrm{I}$ & $\alpha$ & $\delta$ & $\log r$ & $\log \Delta$ \\
\hline April 9 & $12^{\mathrm{h}} 35^{\mathrm{m}} 17^{\mathrm{s}}$ & $-17^{\circ} 24: 2$ & 0.5120 & $0.355^{6}$ \\
\hline 13 & 3221 & 1653.1 & $0.512 \mathrm{I}$ & $0.35^{69}$ \\
\hline 17 & 2935 & $16 \quad 21.3$ & 0.5123 & $0.359^{\circ}$ \\
\hline 21 & 2659 & 1549.1 & 0.5124 & 0.3619 \\
\hline 25 & $243^{8}$ & $15 \quad 16.9$ & $0.5 \times 25$ & 0.3659 \\
\hline 29 & 122232 & -1445.3 & $0.5 \times 26$ & 0.3707 \\
\hline
\end{tabular}

Bei Berechnung der Jupiter- und Saturn-Störungen wurde die Brünnow'sche Methode angewandt und dann folgende Oppositionsephemeride für $\mathrm{I}^{\mathrm{h}}$ M. Z. Berlin berechnet :

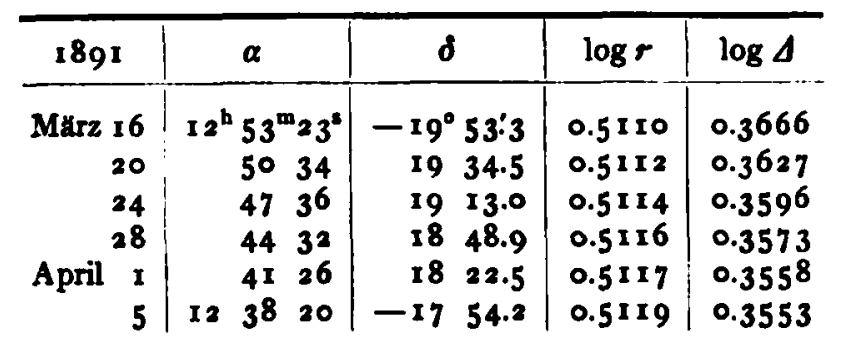

8 in AR. 189 I April I. Oppositionsgrösse $=12.5$.

Prag, böhm. astronomisches Univ.-Institut, I89 I März 12.

Karl l'etr.

\section{Anzeige betr. ein neues General-Register zu den Astr. Nachr.}

Ein General-Register der Astronomischen Nachrichten zu Band 81 bis 120, zusammengestellt von den Herren Dr. H. Kreutz und Dr. R. Schorr ist nahezu im Druck beendigt und wird Anfang April versandt werden können. Der Preis des Bandes ist auf 25 Mark festgestellt. Bestellungen nimmt die Expedition der Astronomischen Nachrichten in Kiel sowie die Buchhandlung von W. Mauke Söhne in Hamburg entgegen.

Kiel I 89 I März 23.

A. Krueger.

In halt zu Nr. 3025. E. C. Pickering. A Fifth Type of Stellar Spectra. 1. - E. C. Pickering. Stars having Peculiar Spectra. New Variable Stars in Aquarius, Delphinus, and Camelopardalus, 5. - F. Plassmann. Zur Theorie von Algol und $\lambda$ Tauri. 5. B. Wanach. Polhöhenbestimmungen am Pulkowaer Passageninstrument im ersten Vertical. 7. - T. Zona. Latitudine di Palermo. 9. - A. Thraen. Genäherte Elemente und Ephemeride des Cometen 1884 III (Wolf). 11. - A. Krueger. Zusatz hierzu. 15. - K. Petr. Ephemeride des Planeten (266) Aline. 15. - A. Krueger. Anzeige betr. ein neues GeneralRegister zu den Astr. Nachr. 15.

Geschlossen 1891 Marz 28 . Horaugeber: A. Krueger. Druck von C. Schaidt. Expedition: Sternwurte in Kiel. 